\title{
Discrimination of structural isomers of amphetamine using carbon dioxide negative-ion chemical ionization mass spectrometry
}

\author{
Kevin B. Thurbide and C.M. Elson* \\ Department of Chemistry, Saint Mary's University, Halifax, N.S., B3H 3C3, Canada \\ Tel.: +1 902420 5653; Fax: +1 902496 8104; E-mail: celson@shark.stmarys.ca \\ P.G. Sim \\ Institute of Marine Biosciences, National Research Council of Canada, 1411 Oxford St., Halifax, \\ N.S., B3H 3Z1, Canada
}

Received 14 August 1996

Revised 2 September 1996

\begin{abstract}
The negative-ion chemical ionization mass spectra of a group of structural isomers of amphetamine have been studied using carbon dioxide as the reagent gas. Characteristic and reproducible differences are observed for each member of the set implying that this technique offers a means of distinguishing among groups of amphetamine isomers. Characteristic adducts to the molecular ion are observed in the form $(\mathrm{M}-[\mathrm{H}]+[\mathrm{O}])$ and $\left(\mathrm{M}-[\mathrm{H}]+\left[\mathrm{CO}_{2}\right]\right)$. Descriptions of some fragments are given based on the mass spectral behaviour of a set of analogue compounds and the results of oxygen-18 labelled carbon dioxide reagent gas experiments. Contents of the carbon dioxide plasma and their impact on various analytes is also discussed.
\end{abstract}

Keywords: Amphetamine, carbon dioxide, chemical ionization, isomers, negative-ion

\section{Introduction}

Over the last decade the trade of illegally prepared 'designer' drugs has been ever growing. Very popular among these substances of abuse are central nervous system stimulants related to amphetamines [1] commonly referred to as 'speed' or 'crack'. The illicit synthesis of these regulated compounds is often accompanied by homologs, precursors, by-products, and isomers of the drug. These can lead to uncertainty in determining the presence of amphetamine in samples and has prompted a need for improved analytical methods to identify it among other structurally related matrix counterparts [2].

${ }^{*}$ Author to whom correspondence should be addressed. 
Various separation methods such as gas and liquid chromatography [3-6], chiral chromatography [7], and capillary electrophoresis [8], have been employed in the determination of different amphetamines from their isomers. While these techniques met with some success, identifications based entirely on retention times are often open to doubt when analogs and isomers elute very closely to one another. Infrared (IR) [6,9], ultra-violet (UV) [9], and nuclear magnetic resonance (NMR) [8] spectroscopies have been used in an attempt to gain more structural information, however, IR and UV spectra of these compounds are very similar and the quantities needed for useful NMR spectra are not always practical. More commonly, tandem methods utilizing mass spectrometry (MS) such as GC-MS [5], and GC-MS-IR [6,10] have also offered a means of differentiating among amphetamine isomers. However, these methods can yield questionable reliability as the electron impact (EI) fragmentation patterns of the isomeric $\mathrm{C}_{3} \mathrm{H}_{8} \mathrm{~N}$ phenyl side chains are virtually indistinguishable from one another and thus do not provide much structural discrimination. Pre-column derivitization of amphetamines has been used to gain more rich and diverse EI fragmentation patterns among mass spectra of the isomers but considerable time can be involved in the pre-treatment steps and the isomers do not always yield stable derivatives that separate well chromatographically [5]. Others have used MS monitoring in the negative ion mode to gain more structural information from their derivatized amphetamines [11,12].

Negative ion chemical ionization (NICI) has been used in mass spectrometry for many years as a means of obtaining more structural information about compounds through specific ion molecule reactions with a variety of reagent gases [13-16]. The use of carbon dioxide in NICI was not very common until the coupling of supercritical fluid chromatography (SFC) with MS inevitably began demonstrating some of its analytical utility [17,18]. Since then carbon dioxide NICI has provided assistance in several demanding applications such as isomer discrimination among nitroso compounds [19] and polycyclic aromatic hydrocarbons [20,21]. While literature precedents of its use remain rather limited, most agree on a common observation of carbon dioxide NICI as being a mildly oxidizing plasma containing an abundance of, if not exclusively, $\mathrm{O}^{-}$[22]. Although the ion source chemistry of the carbon dioxide plasma continues to be somewhat enigmatic, it has proven itself useful for unknown samples as a general ionizing medium when chosen as a NICI reagent gas [23].

This paper describes the discrimination of amphetamine from several of its structural isomers by the use of negative ion chemical ionization employing carbon dioxide as the reagent gas. In addition, some explanations of the fragmentation patterns for each isomer are offered as are descriptions of the ion chemistry affiliated with the carbon dioxide plasma.

\section{Experimental}

All chemicals were reagent grade. The amphetamine isomers were purchased from Aldrich Chemical Company (Milwaukee, WI). The carbon dioxide reagent gas was purchased from Liquid Carbonic Canada (99.99\% purity). The oxygen-18 labelled carbon dioxide was purchased from Isotec Incorporated $(97.2 \%$ purity). A sulphate of the amphetamine drug was acquired through Health Canada and was treated with sodium methoxide to obtain the free methylphenethylamine for examination. The amphetamine side chain isomers are depicted in Fig. 1.

Mass spectra were obtained using a VG Analytical 20-250 quadrupole mass spectrometer (Manchester, UK) which was operated in both the EI $(70 \mathrm{eV})$ and $\mathrm{CI}(100 \mathrm{eV})$ modes. During CI experiments, carbon dioxide filled the ion source which was held at $130^{\circ} \mathrm{C}$ and at a pressure of $4 \times 10^{-4} \mathrm{mBar}$ as indicated on the ion gauge located on the vaccuum manifold between the ion source and the diffusion 
<smiles>CNCCc1ccccc1</smiles>

I<smiles>CCN(C)c1ccccc1</smiles>

III<smiles>CC(CN)c1ccccc1</smiles>

II<smiles>CCNCc1ccccc1</smiles>

IV<smiles>NCCCc1ccccc1</smiles><smiles>CN(C)Cc1ccccc1</smiles>

V

VI<smiles>CC(N)Cc1ccccc1</smiles>

VII

Fig. 1. Structures of the amphetamine isomers investigated in this study. The compounds are: (I) N-methylphenethylamine; (II) $\beta$-methylphenethylamine; (III) N-ethyl-N-methylaniline; (IV) N-ethylbenzylamine; (V) 3-phenyl-1-propylamine; (VI) N,N-dimethylbenzylamine; (VII) amphetamine.

pump. These parameters were deemed to be optimum in terms of signal/noise and selectivity of the plasma to the different isomers.

$\mathrm{N}$-methylphenethylamine and $\beta$-methylphenethylamine were dissolved in methanol to yield $500 \mathrm{ppm}$ solutions and all others were analyzed in their pure forms. Compounds were introduced as $1 \mu \mathrm{l}$ aliquots into the ion source by means of a temperature controlled direct insertion probe inlet on the mass spectrometer.

\section{Results and discussion}

\subsection{Analysis of amphetamine isomers}

Initial investigations were carried out to evaluate the EI mass spectra of the isomers. This was done primarily to detect any discrepancies that may have been present between data collected from our system and literature values. Figure 2 displays the positive ion mass spectra of $\beta$-methylphenethylamine with and without carbon dioxide present $\left(\mathrm{CI}^{+}\right.$in this case is meant only to indicate that the ion source was filled with reagent gas and that positive ions were collected). Not surprisingly, the two spectra are very similar with the exception of lower mass fragments $(\mathrm{m} / \mathrm{z} 44,28)$ owing to the carbon dioxide. The reasonable abundance of a molecular ion $(\mathrm{m} / \mathrm{z} 135)$ and the relatively simpler spectrum 


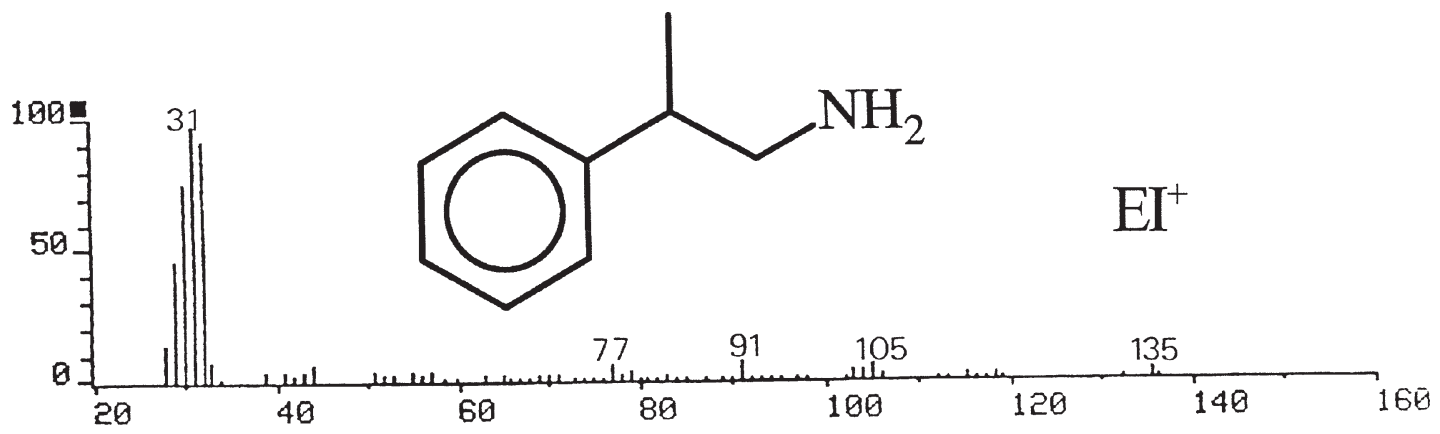

(A)

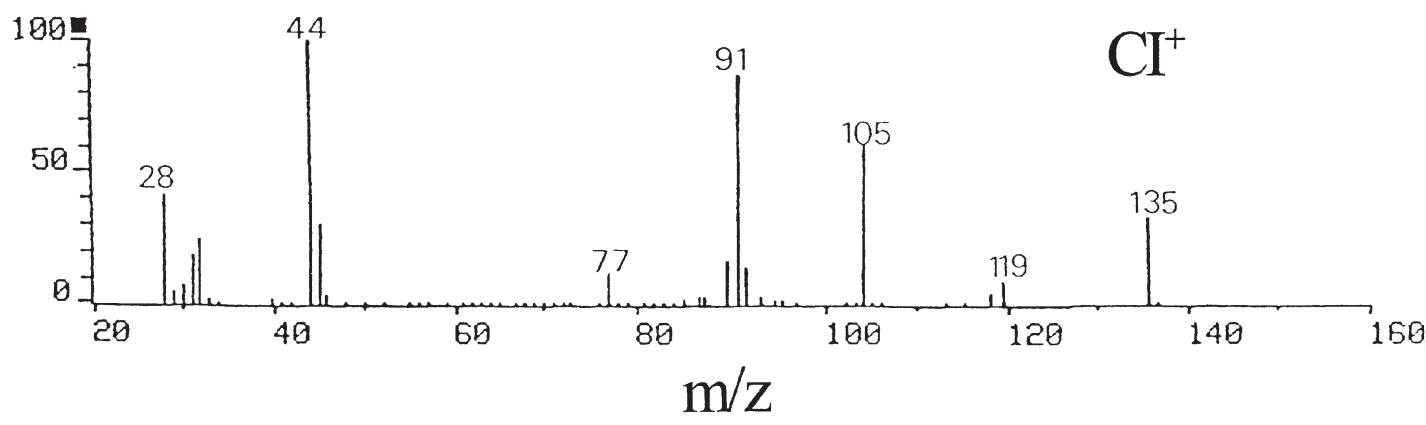

(B)

Fig. 2. Positive ion mass spectra of $\beta$-methylphenethylamine collected under (A) electron impact and (B) charge exchange chemical ionization by carbon dioxide conditions.

obtained by using carbon dioxide seem to be consistent with chemical ionization by charge exchange [13-15,24]. Overall the fragmentations displayed in both spectra are typical of those observed for the other amphetamine isomers which reproduce the pattern of side chain degradation when operated in these modes. The mass spectra in Fig. 2 are also very similar to those previously published [5, 6]. These findings endorse the instrument mass calibration and readdress the original concern of the amphetamine isomers being indiscernible from one another.

When using carbon dioxide CI in the negative ion mode the amphetamine isomers behave considerably different from one another. Figure 3 shows the NICI spectra of all the isomers using carbon dioxide, then oxygen-18 labelled carbon dioxide, and finally that of the amphetamine drug for comparison. Each spectrum is normalized to its most intense peak. All of the fragmentation patterns were found to be very reproducible under these conditions and a quick glance at the figure suggests this method is very effective in discriminating amongst the different amphetamines. While many of the ion fragments and abundances displayed are unique to each isomer there can be drawn a few common observations throughout most of the set.

First is the addition anion of the form $(\mathrm{M}-[\mathrm{H}]+[\mathrm{O}])$ seen at $\mathrm{m} / \mathrm{z} 150$. This has been previously observed in other systems and attests to the mildly oxidizing character of the carbon dioxide plasma [20, $21,24,25]$. The postulation of such an addition is supported by the oxygen-18 labelled carbon dioxide NICI spectra of the isomers. In these conditions the ion at $\mathrm{m} / \mathrm{z} 150$ moves to $\mathrm{m} / \mathrm{z} 152$ indicating the presence of one oxygen atom on the anion. Of the isomers examined, $\beta$-methylphenethylamine, $\mathrm{N}$ - 
$\mathrm{CO}_{2}$
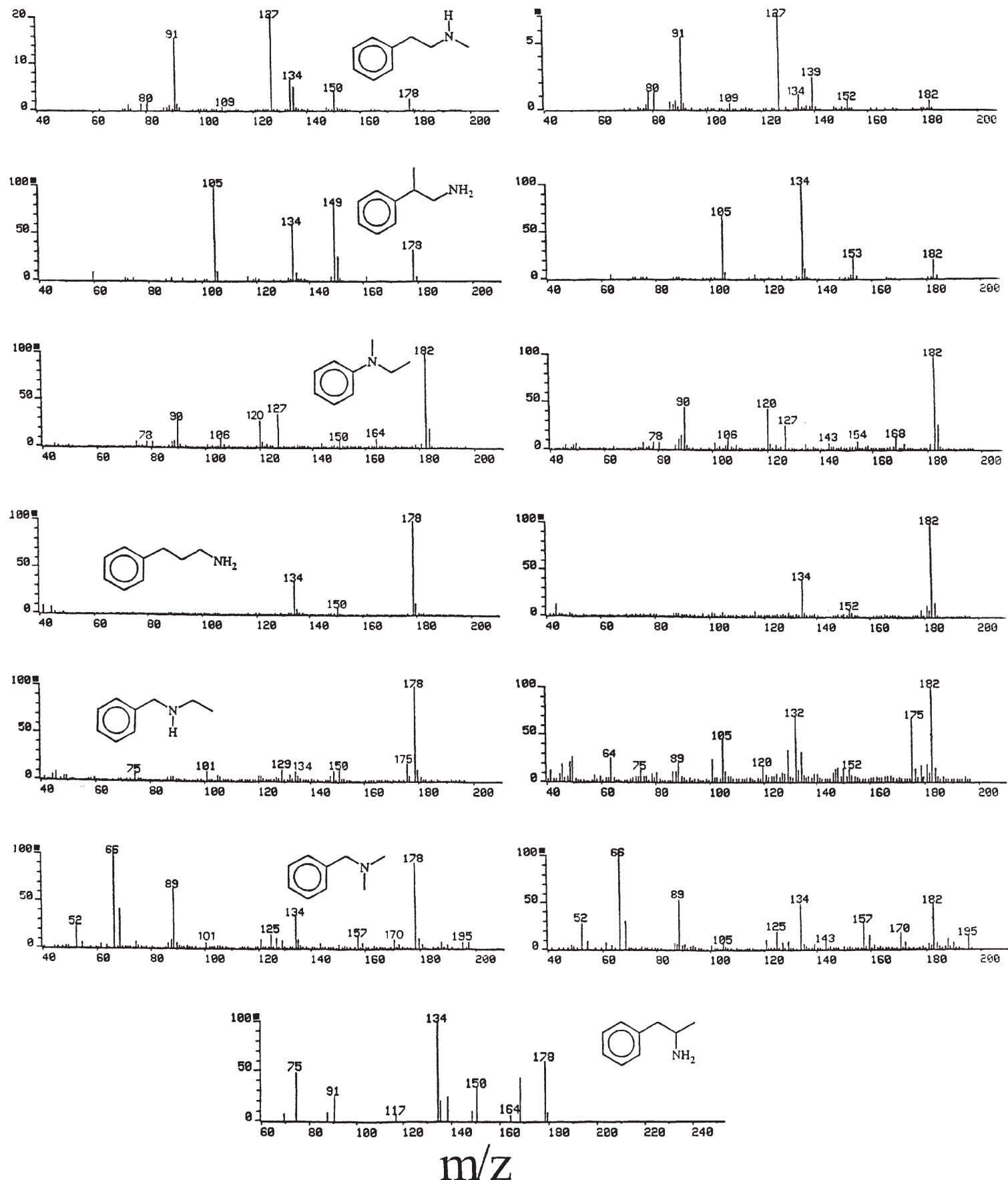

Fig. 3. Negative ion chemical ionization mass spectra of the amphetamine isomers using $\mathrm{CO}_{2}$ and then $\mathrm{C}^{18} \mathrm{O}_{2}$ as the reagent gas. Compounds are as labelled in Fig. 1. The NICI $\mathrm{CO}_{2}$ mass spectrum of the drug amphetamine is shown at the bottom. 
ethyl-N-methylaniline, and N,N-dimethylbenzylamine were the only three that did not yield an anion of this type. This in turn remains analytically useful along with other traits in fingerprinting these isomers' spectra.

Another feature, one that is familiar to all of the spectra, is the addition of carbon dioxide. For all but one spectrum, the anion can be represented by the general form $\left(\mathrm{M}-[\mathrm{H}]+\left[\mathrm{CO}_{2}\right]\right)$ and observed at $\mathrm{m} / \mathrm{z}$ 178. Once again the oxygen-18 labelled carbon dioxide CI spectra support this suggestion as the peak at $\mathrm{m} / \mathrm{z} 178$ moves four mass units to $\mathrm{m} / \mathrm{z} 182$ indicating the presence of two oxygen atoms on the ion. The NICI spectrum for N-ethyl-N-methylaniline is slightly different in that it exhibits carbon dioxide addition accompanied by the loss of an ethyl or methyl group (i.e., $\left(\mathrm{M}-\left[\mathrm{C}_{2} \mathrm{H}_{5}\right]+\left[\mathrm{CO}_{2}\right]\right)$ or $\left.\left(\mathrm{M}-\left[\mathrm{CH}_{3}\right]+\left[\mathrm{CO}_{2}\right]\right)\right)$. These ions also shift from m/z 150 and 164 in the carbon dioxide mode to $\mathrm{m} / \mathrm{z}$ 154 and 168 respectively in the labelled spectrum. It might be argued that the presence of two oxygen atoms on the ion could be a result of the addition of two separate $\mathrm{O}^{-}$ions, however, this would create a doubly charged ion and the peak would be seen at half its nominal mass. Furthermore, it still could not fully account for the attendant increase of 44 mass units. Unfortunately, the obtainable quantities of the regulated amphetamine drug were such that a labelled experiment could not be performed on it. At this point however, the ions at $\mathrm{m} / \mathrm{z} 150$ and 178 seem very likely to be of the forms (M$[\mathrm{H}]+[\mathrm{O}])$ and $\left(\mathrm{M}-[\mathrm{H}]+\left[\mathrm{CO}_{2}\right]\right)$ respectively. Addition of carbon dioxide in NICI has been observed for some nitroso compounds [19]; although less than half of the compounds examined yielded these product anions and all but one gave relative abundances of less than $10 \%$, the authors were able to identify them as being of the type $\left(\mathrm{M}-[\mathrm{H}]+\left[\mathrm{CO}_{2}\right]\right)$. Their notably reduced occurrence of carbon dioxide addition ions relative to our findings could be attributed to the different reagent gas pressure, ion source temperature, and electron energy which they employed or to the chemical nature of the compounds (e.g., the presence of the nitroso groups).

Various other interesting ions were observed at masses greater than the molecular ion (MW 135) but did not shift in the oxygen-18 labelled carbon dioxide mode suggesting that no part of the reagent gas attached itself to the molecule. One prominent example of this is the abundant ion observed at $\mathrm{m} / \mathrm{z}$ 182 in the spectrum of $\mathrm{N}$-ethyl-N-methylaniline. Its mass implies the addition of some other fragment in the ion source, likely a portion of the compound itself to the molecule. If so, it seems reasonable that a dimer of the $\mathrm{C}_{6} \mathrm{H}_{5}-\mathrm{N}$ group would yield an azobenzene anion of moderate stability and fitting mass. An investigation of aniline monitored under the same conditions produced an ion at $\mathrm{m} / \mathrm{z} 182$ which was not the most abundant ion but substantial enough to make a logical assignment of the mass to the dimer structure. In the spectrum of N-ethylbenzylamine the ion at $\mathrm{m} / \mathrm{z} 175$ could also be similar to a dimer of $\mathrm{a}_{6} \mathrm{H}_{5}-\mathrm{C}$ group however this was not fully examined. Ions at $\mathrm{m} / \mathrm{z} 157,170$, and 195 in the spectrum of N,N-dimethylbenzylamine may possibly be adducts of analyte fragments to the molecular ion as well. While their structures are not easily established the compound is air sensitive and the mildly oxidizing plasma conditions would seem conducive to a variety of degradations and additions. The amphetamine drug spectrum has unique ions at m/z 138 and 168 which are believed to be attributable to source contamination that had slowly increased the abundance of these masses in prior experiments of other unrelated compounds.

Some interesting masses appear lower than the molecular ion such as that at $\mathrm{m} / \mathrm{z} 127$ in the spectrum of N-methylphenethylamine. This ion is the most abundant in the spectrum. Given the unusual mass difference, this ion seems as though it is created by eight hydrogen abstractions from the parent molecule resulting in a cyclic aromatic structure resembling isoquinoline. This is supported by the considerable ion abundance at $\mathrm{m} / \mathrm{z} 127$ in the spectrum of isoquinoline under the same conditions. Ions of this type are also seen at m/z 125 and 129 for a few of the other isomers. It should be pointed 
out that this possible cyclization is not consistent throughout the set as 3-phenyl-1-propylamine, which would also be expected to display this behaviour, yields a very simple spectrum with no abstractions of this type at all. Another consideration is that the ion at $\mathrm{m} / \mathrm{z} 127$ is perhaps due to the presence of an iodine impurity in the sample. While the m/z 127 peak in the negative ion mode invites this explanation as a possibility the corresponding $\mathrm{EI}^{+}$and $\mathrm{CI}^{+}$spectra do not offer a trace of evidence of any positive ion species in the same mass range. At present the abundant appearance of such ions is worth noting but more specific experiments (e.g., MS-MS) would be required to fully elucidate their structure. Such an investigation, however, is beyond the analytical boundaries of the current work. One other ion worth discussion is that at $\mathrm{m} / \mathrm{z} 101$ in the spectra of N-ethylbenzylamine and N,Ndimethylbenzylamine which is believed to appear as a result of side chain degradations and hydrogen abstractions producing an ion resembling benzonitrile. This hypothesis was tested by observing the spectra of benzylamine and benzonitrile under the same conditions. Both compounds generated a large abundance of $\mathrm{m} / \mathrm{z} 101$ lending further support to the ion assignment.

Concerning sensitivity, the signals achieved in the $\mathrm{CO}_{2}$ NICI mode are not as large as those obtained in the $\mathrm{EI}^{+}$mode. However, the negative ion spectra do have the advantage of moving ions of interest to higher masses via additions of the reagent gas. In these areas there are fewer ions and this in turn can improve the signal to noise ratio.

\subsection{Negative ion chemistry of the carbon dioxide plasma}

The nature of the reactions involving the carbon dioxide reagent gas are apparently quite diverse and highly dependent upon the conditions inside the ion source as well as the structure of the compound with which it is reacting. For instance, at higher temperatures and pressures in the ion source, the amphetamine isomers produced ions at almost every $\mathrm{m} / \mathrm{z}$ value monitored and background noise was much more prevalent. Spectra obtained under these conditions were practically unusable as they could not be readily interpreted and little discrimination among the set of isomers could be observed. However, as seen in Fig. 3, lower settings of these parameters yield reproducible and unique spectra for each amphetamine isomer. Most likely this is primarily due to the species that are present in the carbon dioxide plasma and their relative reactivity.

Figure 4 exhibits the plasma ions present at different reagent gas pressures of the carbon dioxide. 'Regular' pressure represents that which was used for isomer discrimination as given in the experimental section. The positive ion collection remains essentially the same in both conditions displaying abundant ions at $\mathrm{m} / \mathrm{z} 44,32$, and 28 believed to be $\mathrm{CO}_{2}^{+}, \mathrm{O}_{2}^{+}$, and $\mathrm{CO}^{+}$respectively. The negative ion collection seems to generate entirely $\mathrm{O}^{-}$at $\mathrm{m} / \mathrm{z} 16$ for lower pressures, and predominantly $\mathrm{O}^{-}$at higher pressures along with some other less abundant species (e.g., $\mathrm{CO}_{3}^{-}$at $\mathrm{m} / \mathrm{z} 60$ ). These observations are somewhat expected in view of some other greatly detailed works regarding the ion production of carbon dioxide [26-29]. Interesting though is the isolated appearance of $\mathrm{O}^{-}$in the ion source, when under the same conditions amphetamine isomers have shown the repeated emergence of carbon dioxide adduct ions. The presence of $\mathrm{CO}_{2}^{-}$has been reported in other systems [18], however, its apparent absence from our reagent gas studies would tend to suggest that neutral $\mathrm{CO}_{2}$ adds to the amine anion.

In the context of analytical utility an examination of the type of compounds that are susceptible to similar reactions with the carbon dioxide plasma is warranted. Table 1 gives the results of a general study of a variety of chemical structures and their reactions or lack thereof with the carbon dioxide reagent gas. Again monitored under the same conditions which yield amphetamine isomer 

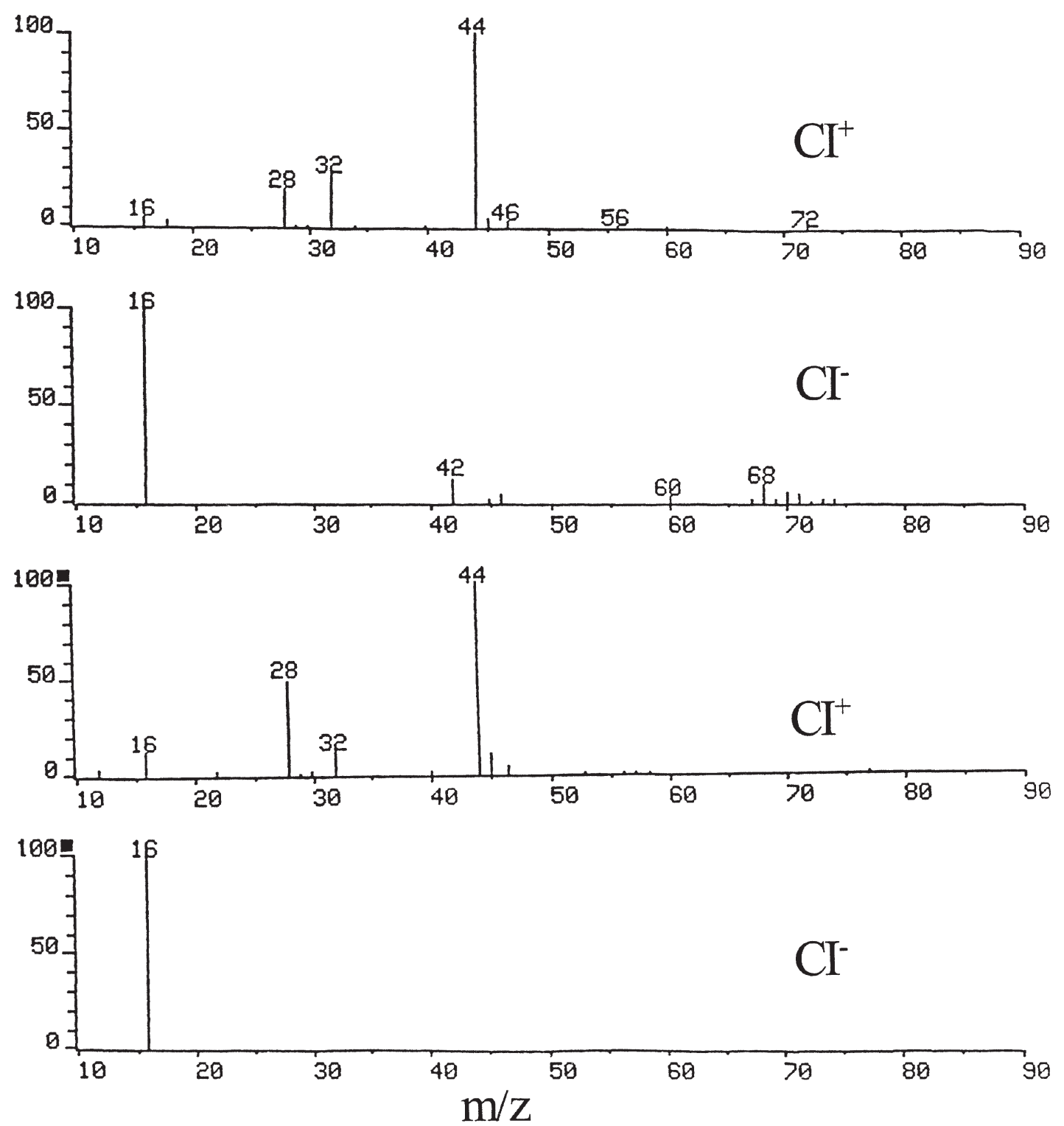

Fig. 4. Analysis of the carbon dioxide plasma under positive and negative ion collection modes at different ion source pressures.

discrimination, the survey displays an assortment of affinities for reaction with the plasma. A cursory glance at the results reveals that a considerable number of the compounds investigated are prone to carbon dioxide addition during chemical ionization and it has already been shown that all of the amphetamine isomers produce these adduct ions. In contrast to previous studies [20,21] oxygen addition is relatively less frequent and more varied in its selectivity. A systematic comparison between the phenylalkylamine structure of the amphetamines and those compounds which also contain some of these components is helpful in gaining an understanding of the plasma's reactivity. This allows certain interesting trends throughout the table to be highlighted and initiates a preliminary guide to the behavioral patterns of the carbon dioxide reagent gas. 
Table 1

Adduct ions that are found in the carbon dioxide NICI mass spectra of a variety of compounds

\begin{tabular}{lll}
\hline Compound & \% Ion abundance & {$\left[\mathrm{CO}_{2}\right]$ addition } \\
\hline N-methylphenethylamine & {$[\mathrm{O}]$ addition } & 15 \\
$\beta$-methylphenethylamine & 20 & 35 \\
N-ethyl-N-metylaniline & - & 10,15 \\
3-phenyl-1-propylamine & - & 100 \\
N-ethylbenzylamine & 10 & 100 \\
N,N-dimethylbenzylamine & 20 & 90 \\
amphetamine & - & 60 \\
benzene & 30 & 20 \\
naphthalene & 65 & 15 \\
isoquinoline & 100 & 30 \\
n-butylbenzene & 100 & 80 \\
sec-butylbenzene & - & 25 \\
tert-butylbenzene & - & 100 \\
benzyl alcohol & - & 100 \\
benzyl amine & - & 90 \\
benzonitrile & - & 10 \\
aniline & - & 25 \\
octane & - & - \\
N-hexylamine & 20 & 50 \\
n-hexadecane & - & - \\
1-hexadecene & - & 100 \\
squalane & - & - \\
squalene & - & - \\
hexatriacontane & - & - \\
nitrobenzene & - & - \\
o-dinitrobenzene & - & - \\
m-dinitrobenzene & - & - \\
chlorobenzene & - & - \\
p-dichlorobenzene & - & - \\
peracetylated & - & \\
\hline
\end{tabular}

For instance the cyclic aromatics benzene, naphthalene, and isoquinoline all undergo oxygen and carbon dioxide addition. While the oxygen adduct ion appears more abundantly among this group, it is not that surprising given the similar behaviour of some other PAH's [20,21]. However, when a side group is attached to the phenyl moiety, as with benzyl alcohol, benzyl amine, benzonitrile, aniline, and the butyl benzenes, oxygen addition ceases while the carbon dioxide adduct ion is abundant in all of their spectra. While it is of general interest that the reactions shift toward carbon dioxide addition when a side group is attached to the aromatic ring, this could also prove to be of significant analytical importance.

Alkyl compounds were also examined but were not generally observed to form any adduct ions with the reagent gas. Exceptions to this are found for n-octane which had a low amount of an $(\mathrm{M}-[\mathrm{H}]+[\mathrm{O}])$ ion present and 1-hexadecene which had an apparently large abundance of carbon dioxide addition to the molecular ion. Also of note $\mathrm{N}$-hexylamine, similar in size to n-octane, produces no oxygen addition but a significant amount of the $\left(\mathrm{M}-[\mathrm{H}]+\left[\mathrm{CO}_{2}\right]\right)$ adduct ion. This may perhaps infer that the double bond character of the former or the amine function in the latter have some implications in the appearance of the carbon dioxide adduct ion. Larger alkyl compounds such as squalane, squalene, $\mathrm{n}$-hexadecane, or hexatriacontane all displayed very rich fragmentation, some at greater $\mathrm{m} / \mathrm{z}$ values than the molecular ion, however, nothing that could be regarded as reagent gas adduct ions. It should be mentioned though that these molecules have 30 or more carbon atoms in their skeleton and do not 
generally yield molecular ions, but do produce many fragments. Hence, any ions corresponding to masses larger than the molecular weight are believed to be intermolecular additions of these fragments.

Finally, largely oxidized or polar compounds such as peracetylated glucose, nitrobenzenes, and chlorobenzenes showed no evidence of any reagent gas addition ions. Although differences were observed between their positive ion EI or CI spectra and the negative ion mode there did not appear to be any adduct ions involving the carbon dioxide plasma. It may be that these compounds are not amenable to further oxidation by the reagent gas or that they have more of an affinity for intermolecular additions (e.g., some $\mathrm{NO}_{2}^{-}$adducts appeared to be present in the mass spectra of the nitrobenzenes).

Thus far a large number of instances of carbon dioxide addition have been shown as well as some affinity patterns for this addition. One final point of interest is the location of carbon dioxide addition to these molecules. In some instances, such as $\mathrm{N}$-methylphenethylamine and $\beta$-methylphenethylamine where the phenyl and amine groups are separated by two carbon atoms, the carbon dioxide appears to be attached to the benzyl moiety. This is supported by oxygen-18 labelled carbon dioxide spectra which show some fragment ions, due to losses of the side chain, to also shift four mass units indicating that carbon dioxide was still bound to the molecule while the alkylamine portion was degrading. It is also possible that it attaches to the phenyl group, although the position is unclear. For N-ethylbenzylamine and N,N-dimethylbenzylamine, where the phenyl and amine groups are separated by one carbon atom, the $\mathrm{CO}_{2}$ appears bound to the amine moiety. This is supported by the ion at $\mathrm{m} / \mathrm{z} 101$, due to the loss of the phenyl group (77 mass units) from the m/z 178 ion, which also shifts four mass units in the labelled reagent gas experiment. Other isomers such as N-ethyl-N-methylaniline seem to indicate the exchange of carbon dioxide for the ethyl or methyl group. If this variance can be attributed to the number of carbon atoms separating the amine function from the phenyl group then it would help to predict where the $\mathrm{CO}_{2}$ will attach to the molecule.

\section{Conclusion}

The use of carbon dioxide as a reagent gas in the negative ion mode has been shown to yield unique and reproducible mass spectra for each in a series of amphetamine structural isomers. Their characteristic behaviour under these conditions efficiently produced abundant and richly fragmented spectra. The simple and fast procedure suggests usage of this technique for fingerprinting and identifying these compounds in complex matrices. This should enable the analyst to discard the uncertainties encountered when using electron impact mass spectra in these applications and avoid additional derivatization steps often employed for isomer discrimination.

Formation of oxygen adducts are witnessed for some of the amphetamine isomers and various analog compounds, where carbon dioxide addition is observed in nearly every case. Location of this addition varies between the benzyl moiety and the alkylamine moiety depending on the compound under investigation and is postulated to vary with the number of carbon atoms separating the phenyl and amine groups. The contents of the reagent gas under these conditions is found to be predominantly $\mathrm{O}^{-}$.

\section{Acknowledgements}

The authors gratefully acknowledge the assistance of Dr R.K. Boyd and the National Research Council of Canada throughout the course of this work. 


\section{References}

[1] M.A. Schuckit, Drug and Alcohol Abuse: A Clinical Guide to Diagnosis and Treatment, Ch. 5, Plenum Medical Book Company, New York, 1990.

[2] W.H. Soine, Med. Res. Rev. 6 (1986), 41.

[3] M. Longo, C. Martines, L. Rolandi and A. Cavallaro, J. Liq. Chromatogr. 17 (1994), 649.

[4] K. Hayakawa, N. Imaizumi, H. Ishikura, E. Minogawa, N. Takayama, H. Kobayashi and M. Miyazaki, J. Chromatogr. 515 (1990), 459.

[5] C. Randall Clark, J. DeRuiter, A.K. Valaer and F. Taylor Noggle, J. Chromatogr. Sci. 33 (1995), 485.

[6] W. Duncan and W.H. Soine, J. Chromatogr. Sci. 26 (1988), 521.

[7] T. Nagai and S. Kamiyama, J. Chromatogr. Biomed. Appl. 525 (1990), 203.

[8] S.K. Branch, U. Holzgrabe, T.M. Jefferies, H. Mallwitz and M.W. Matchett, J. Pharm. Biomed. Anal. 12 (1994), 1507.

[9] F. Taylor Noggle, C. Randall Clark, K.H. Bouhadir and J. DeRuiter, J. Chromatogr. Sci. 29 (1991), 31.

[10] E. Meyer, J.F. Van Bocxlaer, W.E. Lambert, L. Thienpont and A.P. De Leenheer, J. Anal. Toxicol. 20 (1996), 116.

[11] H.J. Leis, W. Windischhofer and R. Wintersteiger, Biol. Mass Spectrom. 23 (1994), 637.

[12] M.L.J. Reimer, O.A. Mamer, A.P. Zavitsanos and A. Waheed Siddiqui, Biol. Mass Spectrom. 22 (1993), 235.

[13] R.C. Dougherty, Anal. Chem. 53 (1981), 625A.

[14] H. Budzikiewicz, Mass Spectrom. Rev. 5 (1986), 345.

[15] J.H. Bowie, Mass Spectrom. Rev. 9 (1990), 349.

[16] L.J. Sears and E.P. Grimsrud, Anal. Chem. 61 (1989), 2523.

[17] R.D. Smith, H.R. Udseth and H.T. Kalinoski, Anal. Chem. 56 (1984), 2971.

[18] E.C. Huang, B.J. Jackson, K.E. Markides and M.L. Lee, J. Microcolumn Sep. 2 (1990), 88.

[19] O. Suzuki and H. Brandenberger, Fresenius' Z. Anal. Chem. 323 (1986), 217.

[20] P.G. Sim and C.M. Elson, Rapid Commun. Mass Spectrom. 2 (1988), 137.

[21] C.M. Elson and P.G. Sim, Rapid Commun. Mass Spectrom. 4 (1990), 37.

[22] H. Brandenberger, Recent Dev. Mass Spectrom. Biochem. Med. 6 (1980), 391.

[23] H. Brandenberger, Int. J. Mass Spectrom. Ion Phys. 47 (1983), 213.

[24] A.G. Harrison, Chemical Ionization Mass Spectrometry, 2nd. ed., CRC Press, Boca Raton, FL, 1992.

[25] E.A. Stemmler and M.V. Buchanan, Org. Mass Spectrom. 24 (1989), 705.

[26] R. Locht, M. Davister, W. Dezer, H.W. Jochims and H. Baumgärtel, Chem. Phys. 138 (1989), 433.

[27] C.D. Cooper and R.N. Compton, Chem. Phys. Lett. 14 (1972), 29.

[28] G.J. Schulz, Phys. Rev. 128 (1962), 178.

[29] J.G. Dillard, Chem. Rev. 73 (1973), 617. 


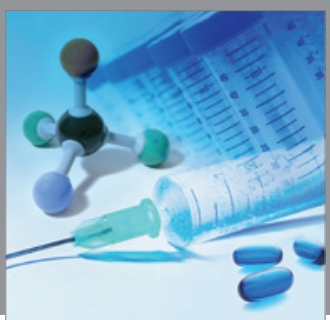

International Journal of

Medicinal Chemistry

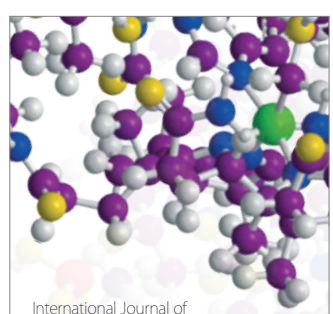

Carbohydrate Chemistry

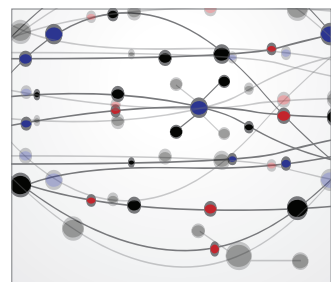

The Scientific World Journal
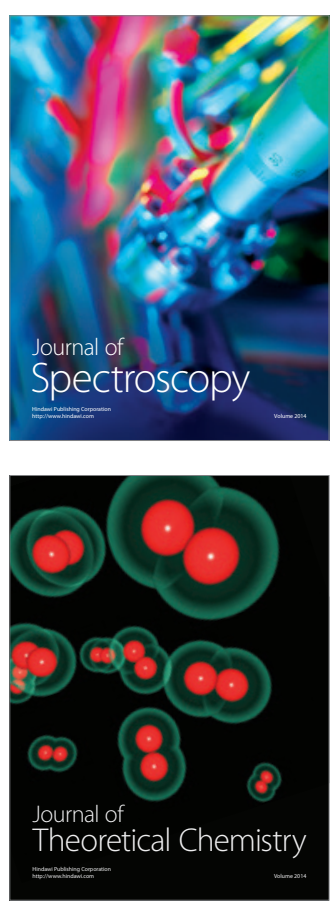
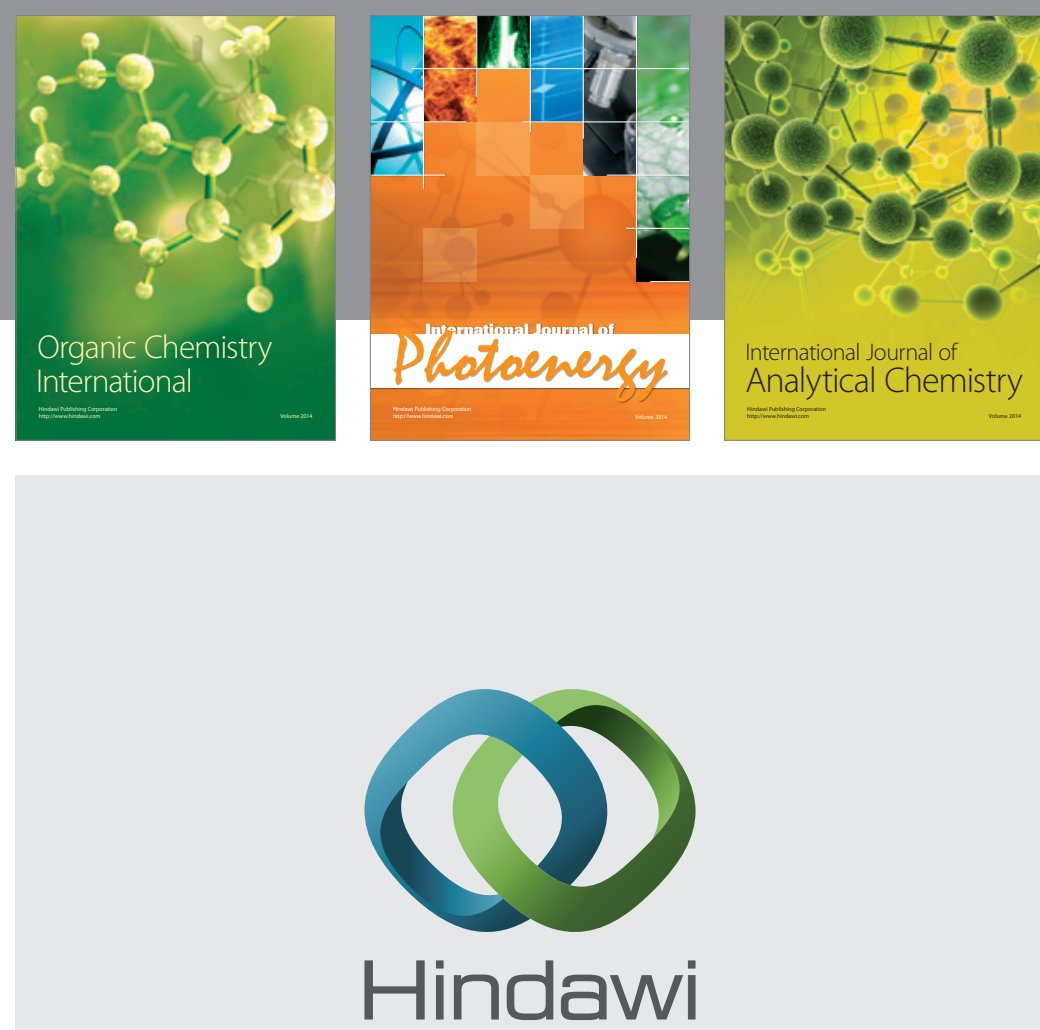

Submit your manuscripts at

http://www.hindawi.com
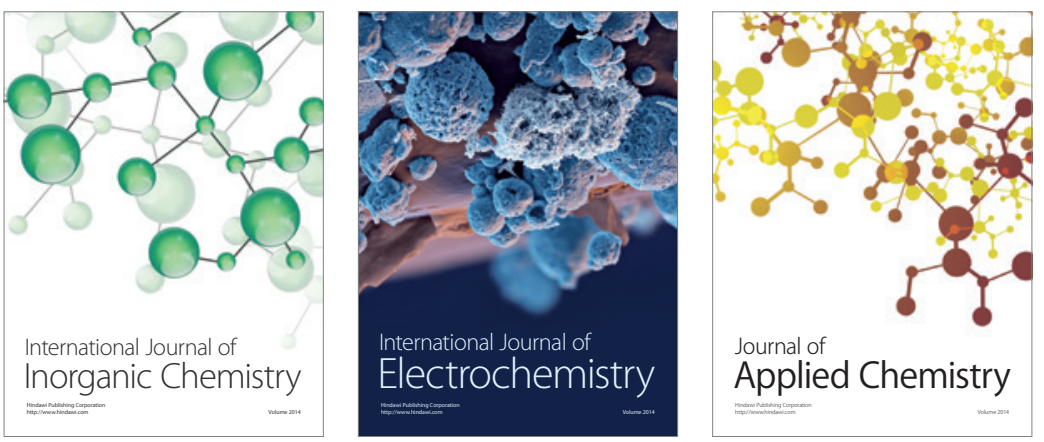

Journal of

Applied Chemistry
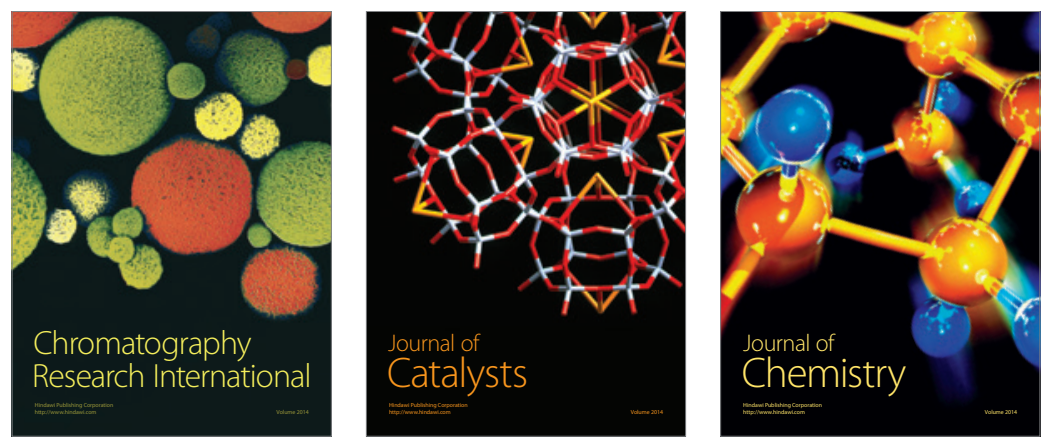
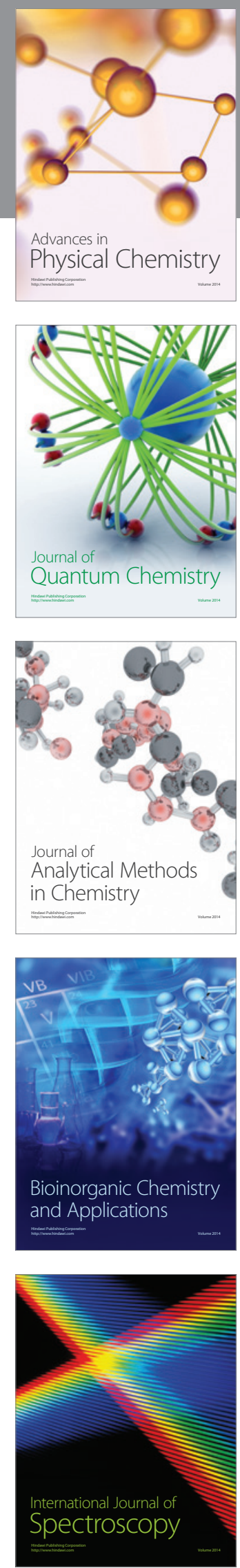